\title{
Mental Illness
}

\section{Sergio Machado ${ }^{1,2}$}

${ }^{1}$ Intercontinental Neuroscience Research Group, Laboratory of Panic and Respiration, Institute of Psychiatry Federal University of Rio de Janeiro (IPUB/UFRJ), Rio de Janeiro, Brazil

${ }^{2}$ Physical Activity Neuroscience Laboratory, Physical Activity Sciences Postgraduate Program, Salgado de Oliveira University (UNIVERSO), Niterói, Brazil

\section{Editorial}

Mental illness and its possible cure have been documented in 'Charaka Samhita, which was written in sixth century BC in India. There are several other documentary evidences are also available, from which concept of mental illness in ancient world is evident. However, with progress of time and research, distinct classification of brain related illness has been presented and 'psychiatry' is one of those specialized branch. The term 'psychiatry' was coined in 1808 by Johann Christian Reil, a German physician. It literally means treatment of the soul. As Psychiatry has two interacting aspects-biological and social, psychiatric disorders pose a high socio-economic burden. The high treatment costs as well as loss of productivity add to the costs incurred. The Journal of Psychiatry presents leading edge research in all disciplines of Psychiatry such as behavior, cognition, and neurological problems. The current issue of the Journal of Psychiatry features some interesting scientific findings. Bedaso et al. [1] observed a positive correlation between age, social stigma and anxiety in patients undergoing Antiretroviral Therapy (ART) at Hawassa Universty Referral Hospital, Ethiopia. They also observed that female patients were 8.2 times susceptible to anxiety as compared to men, and that lower CD4 counts correlated with high anxiety. Li et al. [2] found that severe metabolic disturbances are associated with recurrent depression as opposed to acute depression in the rat model of depression. Luzny Jan [3] identified that Hypodermoclysis is a feasible procedure for replacing fluid in terminally ill elderly patients suffering from dehydration. Girma et al. [4] observed that depression is highly prevalent in elderly individuals, followed by women, illiterate people, unmarried people, chronically ill people, and people having cognitive impairments. Adefalu et al. [5] observed that disclosure of a child's HIV status did not correlate with development of psychiatric disorders. Megan Buoey [6] has authored a report regarding the improvements needed in the current psychiatric system with respect to the use of psychiatric drugs for treating schizophrenia.

Anxiety is a vague feeling of uneasiness, apprehension, and tension, which manifests as irrational avoidance of situations or objects. Reports suggest that anxiety is highly prevalent in HIV patients; its frequency of prevalence in these patients is roughly three times higher than in normal individuals. More often than not, the anxiety and depression coexist in HIV patients. Bedaso et al. [1] investigated the prevalence of anxiety and associated factors among patients undergoing Antiretroviral Therapy (ART) at Hawassa Universty Referral Hospital, Ethiopia. The results revealed that $17.4 \%$ of the affected patients suffered from anxiety. A positive correlation between age and anxiety was also revealed by the study. Women exhibited higher incidence of anxiety (8.2 times higher) as compared to men. Patient facing social stigma were 2.7 times more likely to be anxiety prone. Surprisingly, CD4 counts were also associated with prevalence of anxiety; people having CD4 count $<500$ cells $/ \mathrm{ml}$ were 2.6 times more prone to have anxiety.

Recurrent depression imposes a high cost on the patient as well as the economy. Identification of biomarkers of recurrent depression will help prevent the disease. Li et al. [2] exposed a rat model of depression, to chronic unpredicted mild stress in order to induce depression.
The rat serum was collected and subjected to metabolomics using gas chromatography/time-of-flight mass spectrometry. The authors observed that serum Oleinic acid and Palmitinic acid levels were low during the onset phase, while serum levels of alanine, cholesterol, 6-Desoxy-Mannopyranose, oleinic acid, and stearic acid were decreased in recurrent depression. Thus, more severe metabolic disturbances are observed in recurrent depression as opposed to acute depression. Thus, the paradigm of recurrent depression may provide insights into the potential biomarkers for depression.

Senior citizens are more vulnerable to dehydration as it may lead to more confusing state of mind, disturbed ionic homeostasis, higher risk of infection and cardiac failure. Different modes of fluid replenishment are available: intravenous therapy, peroral intake, enteral stomach tube, nasogastric tube rehydration, or hypodermoclysis (subcutaneous infusions). Jan [3] investigated the side effects of hypodermoclysis in elders with terminal phase dementia. Towards this, the authors conduced an Open-label trial with patients with terminal dementia $(\mathrm{n}=48)$, and subjected them to hypodermoclysis for rehydration. Interviews were conducted to evaluate the complications associated with hypodermoclysis. The results revealed that Hypodermoclysis was well tolerated by the patients; the process was easy, efficient, and caused minimum discomfort to the patients. Auxiliary side-effects such as: local infection, local erythema, and local edema, were rare. Thus, Hypodermoclysis can be considered to be a convenient procedure for fluid replacement in terminally ill elderly patients suffering from dehydration.

Depression represents one of the most profound health problems currently facing the global healthcare. Depression is estimated to be responsible for $5.7 \%$ of the total disease burden in 2020. Though not age specific, it is the most predominant mental health disorder in the elderly Girma et al. [4], investigated the prevalence of depression among the elderly in Harar, Ethiopia. Towards this end, a community based crosssectional study was conducted $(\mathrm{n}=352)$, where the Geriatric depression rating scale (GDS-15) was employed to assess depression. Respondents with a score of 5 and above on the GDS-15 scale were considered as depressed. The authors observed that depression was prevalent in $28.5 \%$ of the elderly population. Females, illiterate people, incomplete education, unmarried people, chronically ill people, and people with cognitive impairments exhibited high prevalence of depression.

*Corresponding author: Sergio Machado, Physical Activity Neuroscience Laboratory, Physical Activity Sciences Postgraduate Program, Salgado de Oliveira University (UNIVERSO), Niterói, Brazil, Tel: +552121384942; E-mail: secm80@ gmail.com

Received: May 04, 2017; Accepted: May 04, 2017; Published: May 15, 2017

Citation: Machado S (2017) Mental IIIness. J Psychiatry 20: e109. doi:10.4172/23785756.1000e109

Copyright: () 2017 Machado S. This is an open-access article distributed under the terms of the Creative Commons Attribution License, which permits unrestricted use, distribution, and reproduction in any medium, provided the original author and source are credited 
Acquired immune deficiency Syndrome (HIV/AIDS) is associated with a high rate of social stigma and discrimination. As a result, parents of children with the disease do not divulge the child's HIV positive status. Disclosure of HIV status to children is a particularly difficult task for parents or caregivers. The reports concerning the link between disclosure and psychiatric morbidity in HIV positive children are inconclusive, with some studies reporting that children who were aware of their HIV positive status had more psychiatric problems while others indicate that disclosure might actually contribute positively in terms of their psychological wellbeing. Adefalu et al. [5] assessed the pervasiveness of disclosure, the children's awareness of their HIV status, and the effect of disclosure on the mental health of HIV-infected children at the HIV clinic of the University of Ilorin Teaching Hospital, Nigeria (UITH). The authors observed that disclosure of a child's HIV status did not result in development of psychiatric disorders. However, the authors claim that the role of socioeconomic and demographic factors needs to be considered for any such study in the future.

Buoey [6] has written a report concerning the reasons improvements are needed in the current psychiatric system with respect to the use of psychiatric drugs and how they may be insufficient for treating schizophrenia. The author focusses on higher success rates of alternative, minimum medication strategies, like open dialogue, which focus on psychotherapies, as opposed to the conventional antipsychotic drugs. The author shares her opinion regarding the fundamental flaws with the current viewpoints on schizophrenia. The author campaigns for a new strategy for Schizophrenia treatment, involving the integration of psychotherapies and lowering the dosage of antipsychotics.

\section{References}

1. Bedaso A, Belagavi D, Bekele G, Mekonnen N (2016) Factors Associated with Anxiety Disorder among ART Clients attending Antiretroviral Therapy Clinic at Hawassa University Referral Hospital, Hawassa, SNNPR, Ethiopia. J Psychiatry 20: 394.

2. Li Z, Yang S, Zhu C, Chen J, Ding X, et al. (2017) The GC/TOFMS based Serum Metabolomics in Rats with Depression-like Behavior after Exposure and Re-exposure to Chronic Unpredictable Mild Stress. J Psychiatry 20: 396.

3. Jan $L$ (2017) The Hypodermoclysis - Comfortable Way to Rehydration in Patients with End-Stage Dementia. J Psychiatry 20: 397.

4. Girma M, Hailu M, Wakwoya A, Yohannis Z, Ebrahim J (2016) Geriatric Depression in Ethiopia: Prevalence and Associated Factors. J Psychiatry 20: 400.

5. Adefalu MO, Florence M, Ayinmode T, Issa BA, Adefalu AA (2016) Does Disclosure of HIVIAIDS Status to Children with HIVIAIDS Affect their Mental Health? J Psychiatry 20: 399.

6. Buoey M (2016) The Role of Psychiatric Drugs and their Minimal-Medication Alternatives in the Treatment of Schizophrenia. J Psychiatry 20: 395. 